\title{
Controle glicêmico e lipídico de pacientes com diabete tipo 2 em tratamento combinado de metformina e insulina
}

\author{
Glycemic and lipid control of patients with type 2 diabetes in combined \\ treatment of metformin and insulin \\ Fábio Brasili ${ }^{1}$, Andreia Mara Brolezzi Brasil²
}

\begin{abstract}
Palavras-chave:
Metformina

Insulina

Lipídios

Diabetes Mellitus

\section{Resumo}

Este é um estudo transversal em que foram avaliados os dados laboratoriais de perfil lipídico e hemoglobina glicada (HbA1c) dos 105 pacientes portadores de diabetes mellitus tipo 2 em uso de insulina na área adstrita da Unidade de Saúde São Paulo do Programa de Saúde da Família. Teve como objetivo identificar possíveis variações nesses parâmetros quando associada metformina ao tratamento. Desses pacientes, 33 foram excluídos por apresentarem vieses. Dos restantes, 44 estavam em tratamento combinado de metformina e insulina - estes apresentaram níveis de HbA1c e triglicerídeos significativamente inferiores ao grupo controle em tratamento isolado com insulina (n=28); não se observaram diferenças de significado estatístico com relação ao colesterol total e HDL. 0 estudo sugeriu que, pela disponibilidade gratuita da metformina para a população, tal associação deveria ser considerada como primeira escolha em saúde pública.
\end{abstract}

Key Words:

Metformin

Insulin

Lipids

Diabetes Mellitus

\section{Abstract}

This transverse study was undertaken to assess serum lipoprotein cholesterol, glycated hemoglobin ( $\mathrm{HbA1C}$ ) and triglyceride levels of 105 patients with type 2 diabetes mellitus in insulin use belonging to a health care area of the Family Health Program (FHP). It had as objective to identify possible variations in those parameters when metformin is associated to the treatment. Thirty-three of those patients were excluded by presenting inclinations. Of the remaining, 44 subjects were on combination therapy of metformin and insulin - these indicated serum levels of $\mathrm{HbA1C}$ and triglycerides significantly lower than the control group in treatment with insulin alone $(\mathrm{n}=28)$; statistical differences were not observed regarding high-density lipoprotein and total cholesterol levels. The research appeared that, due to free readiness of metformin for the population, combined therapy should be used as first choice in public health.

\footnotetext{
Médico, preceptor do Estágio em Medicina de Família e Comunidade da Pontifícia Universidade Católica do Paraná (PUC/PR), Curitiba (PR), Brasil.

${ }^{2}$ Nutricionista, especialista em Saúde Coletiva, Curitiba (PR), Brasil.

Endereço para correspondência: Fábio Brasil / Andreia Mara Brolezzi Brasil, Rua Irmão Luiz Vicente, 269 - Cajuru, CEP: 82900-140 Curitiba, PR, Brasil, E-mail: fabio.brasi|@pucpr.br
} 


\section{Introdução}

A diabetes mellitus tipo 2 (DM2) é uma síndrome heterogênea que resulta de deficiência na secreção de insulina pelas células- $\beta$ do pâncreas ou de hiperglicemia por aumento da resistência à insulina nos tecidos periféricos ${ }^{1}$; está associada ao desenvolvimento de complicações crônicas microvasculares e macrovasculares de elevada morbidade e mortalidade.

Dislipidemia costuma estar relacionada à diabete e caracteriza-se por níveis baixos de lipoproteínas de alta densidade (HDL, do inglês high-density lipoprotein) e valores altos de triglicerídeos, lipoproteínas de baixa densidade (LDL, do inglês low-density lipoprotein) e colesterol total ${ }^{2}$. Hipercolesterolemia é causa direta de eventos cardíacos isquêmicos, sendo que o não-HDL colesterol, colesterol total menos HDL, se mostrou como melhor previsor de mortalidade cardiovascular que o $\mathrm{LDL}^{3}$.

Os triglicerídeos são um fator de risco independente para doenças cardiovasculares e há necessidade de baixar seus níveis no plasma; depois do ajustamento do HDLcolesterol, esses riscos abrandaram, ainda assim, assumindo importância estatística ${ }^{4}$.

As estatinas são drogas de primeira linha no tratamento das dislipidemias, sendo que sua associação com fibratos mostrou benefícios adicionais ${ }^{5}$; como alternativas: niacina, colestiramina, ezetimiba e orlistate ${ }^{6}$.

A hemoglobina glicada (HbA1c) é o principal determinante para avaliação do controle glicêmico em pacientes portadores de diabetes mellitus; foi demonstrado, pelo importante estudo United Kingdom Prospective Diabetes Study (UKPDS) ${ }^{7}$, que manter seu nível abaixo de $7 \%$ reduz significativamente o risco de desenvolvimento das complicações microvasculares da doença.

Os níveis de $\mathrm{HbA1c}$ não se alteram imediatamente após a normalização da glicose no sangue; o intervalo para que atinja os parâmetros desejados depende do tempo de vida dos eritrócitos. Portanto, o exame para avaliar a eficácia de um tratamento deve ser realizado aproximadamente três meses após seu início ou modificação ${ }^{8}$.

Quando dieta, exercícios físicos e, eventualmente, perda de peso não forem suficientes para a normalização da glicemia, utilizam-se medicamentos por via oral'; sendo que, no UKPDS, a metformina foi o único desses fármacos que determinou uma diminuição expressiva da incidência de complicações macrovasculares em pacientes obesos ou com sobrepeso, os quais representam a maioria absoluta dos diabéticos tipo $2^{10}$, a ponto de os últimos consensos (ADA e ESDA) a indicarem como terapia inicial junto com as mudanças de estilo de vida ${ }^{11}$. Esse medicamento é derivado das biguanidas, substâncias que potencializam a ação insulínica pelo aumento do número de receptores periféricos, diminuição da produção hepática de glicose e da sua absorção intestinal ${ }^{6}$.
Em consenso, recomenda-se iniciar insulina exógena nos casos de hiperglicemia severa ou quando ocorre falência do tratamento com fármacos orais e mudanças do estilo de $v^{\text {vida }}{ }^{11}$. As combinações terapêuticas de insulina com os hipoglicemiantes orais têm sido utilizadas cada vez mais em virtude de potencialmente reduzir sua dosagem e também obter melhor controle da glicemia, possibilitando maior receptividade por parte do paciente ${ }^{12}$.

O Sistema Único de Saúde (SUS) disponibiliza gratuitamente, de rotina, apenas dois hipoglicemiantes orais, que são os mesmos disponíveis na Farmácia Popular do Brasil: metformina e glibenclamida ${ }^{13}$, sendo que, quando associado à insulina, o primeiro apresenta vantagens; em relação ao outro, quanto ao controle de peso, melhoria do controle glicêmico e menor risco de hipoglicemia ${ }^{14}$.

O presente artigo teve como meta comparar a associação de insulina e metformina contra insulina isolada, analisando os valores laboratoriais de hemoglobina glicada e perfil lipídico de rotina (colesterol total, HDL e triglicerídeos), em uma amostra de prontuários médicos de portadores de DM2, em uma unidade de saúde da família.

\section{Metodologia}

Este é um estudo com delineamento transversal, em que foram avaliados os dados laboratoriais de hemoglobina glicada e perfil lipídico de todos os 105 portadores de DM2 em uso de insulina na área adstrita da Unidade de Saúde São Paulo do Programa de Saúde da Família (PSF), localizada no município de Curitiba, Paraná. Os hábitos culturais e alimentares são relativamente homogêneos.

Desses resultados, 33 indivíduos apresentaram algum critério de exclusão, a saber: início da terapia com insulina ou metformina há menos de três meses, uso de qualquer fármaco hipoglicemiante que não seja metformina, utilização de medicamentos para controle de perfil lipídico, como estatinas e orlistate, ou dados laboratoriais incompletos.

Restaram 72 análises de pacientes, as quais foram divididas em 2 grupos: aqueles que estavam em tratamento combinado de insulina e metformina $(n=44)$ e aqueles que estavam em tratamento isolado com insulina $(n=28)$. Os dados foram coletados por meio de pesquisa em prontuário eletrônico no mês de fevereiro de 2009, levando em consideração os valores laboratoriais mais recentes.

Os cálculos estatísticos foram efetuados com base no software BioEstat 5.0; os valores das variáveis contínuas foram expressos em média e desvio padrão, sendo utilizado na sua comparação o teste $t$ de Student. O nível de significância estabelecido foi 0,05 . 


\section{Resultados}

A dose média de insulina (NPH+regular) no grupo que não utilizava metformina foi de 56,56 unidades/dia; no grupo que utilizava, 49,92 unidades/dia.

Os indivíduos que estavam em tratamento isolado com insulina, quanto aos níveis séricos de $\mathrm{HbA1c}$, apresentaram $9,66 \pm 2,15 \%$. Naqueles que estavam em terapia combinada com metformina, os níveis situaram-se entre $8,87 \pm 1,77 \%$. As diferenças foram estatisticamente significativas $(\mathrm{p}<0,05)$ (Figura 1).

$\mathrm{Na}$ Figura 2 estão representados os níveis séricos de colesterol total; nos pacientes em uso de insulina isolada, $210,1 \pm 38,8 \mathrm{mg} / \mathrm{dL}$. Com terapia associada ao hipoglice-

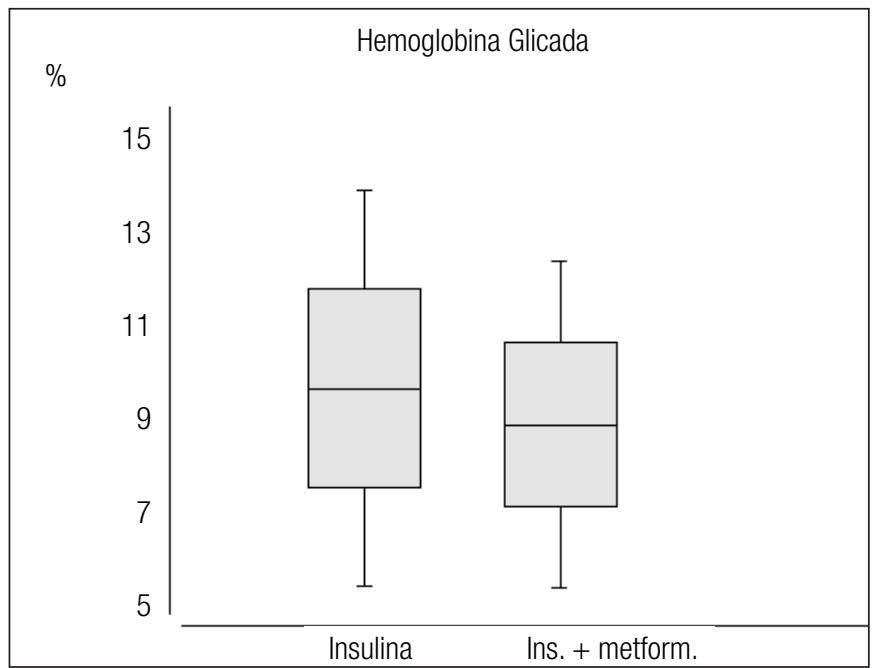

Figura 1: Níveis de hemoglobina glicada dos pacientes diabéticos tipo 2 em tratamento isolado com insulina e tratamento combinado de insulina+metformina.

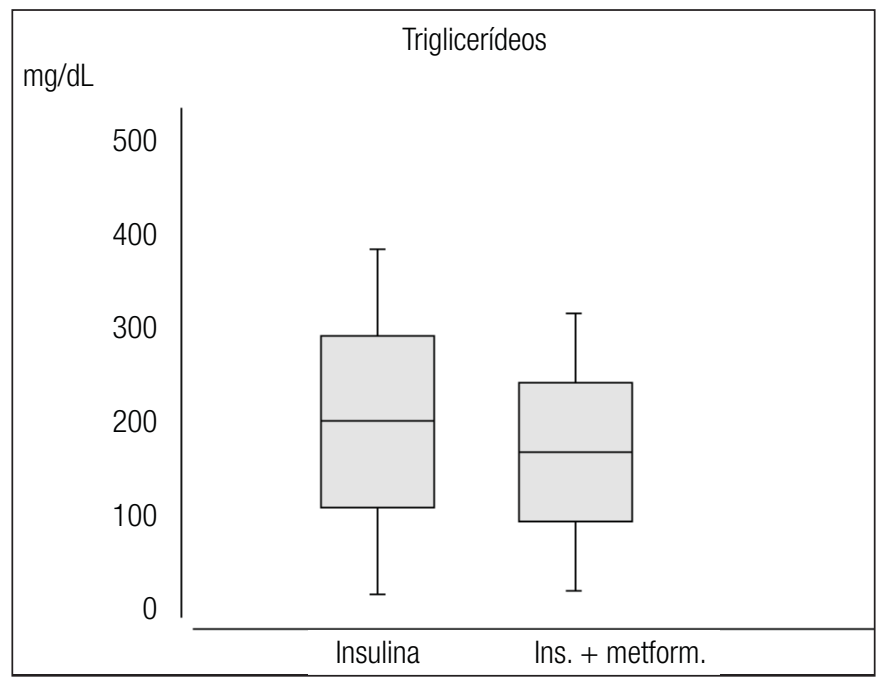

Figura 4: Níveis de triglicerídeos dos pacientes diabéticos tipo 2 em tratamento isolado com insulina e tratamento combinado de insulina+metformina. miante oral, observou-se $202,3 \pm 42,2 \mathrm{mg} / \mathrm{dL}$. As diferenças não foram estatisticamente significativas $(\mathrm{p}>0,05)$.

A Figura 3 demonstra que os dados encontrados, no tratamento isolado com insulina, referentes aos níveis séricos de $\mathrm{HDL}$, registraram $44,7 \pm 10,2 \mathrm{mg} / \mathrm{dL}$. Nos portadores de diabete em terapia combinada com metformina, obteve-se $47 \pm 12,7$ $\mathrm{mg} / \mathrm{dL}$. As diferenças não foram estatisticamente significativas $(\mathrm{p}>0,05)$.

Observa-se, na Figura 4, que os níveis séricos de triglicerídeos, nos indivíduos em tratamento isolado com insulina, foram de $200 \pm 93,3 \mathrm{mg} / \mathrm{dL}$. Nos usuários de metformina e insulina, registrou-se $166,8 \pm 74,7 \mathrm{mg} / \mathrm{dL}$. As diferenças foram estatisticamente significativas $(p<0,05)$.

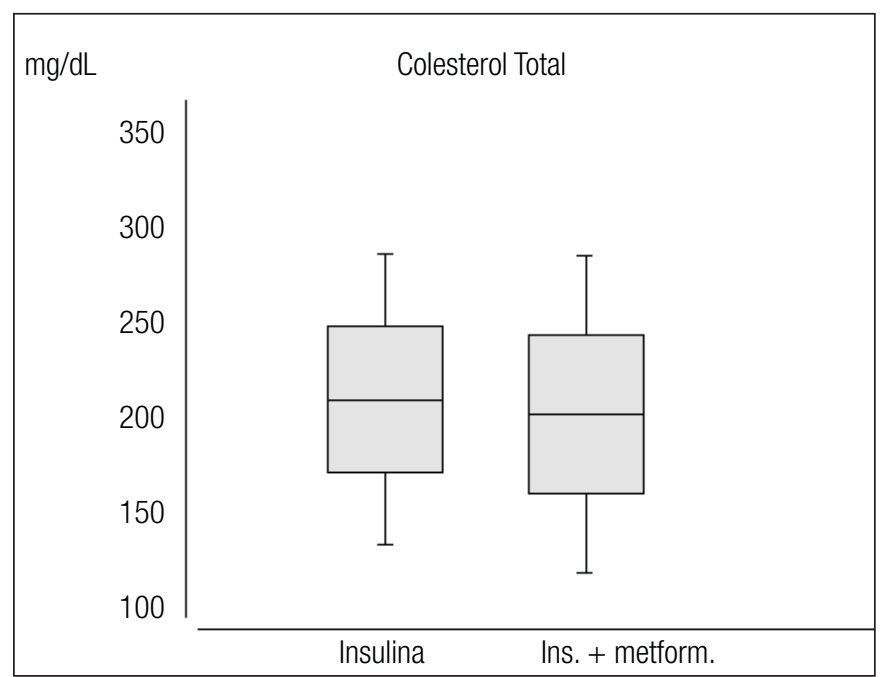

Figura 2: Níveis de colesterol total dos pacientes diabéticos tipo 2 em tratamento isolado com insulina e tratamento combinado de insulina+metformina.

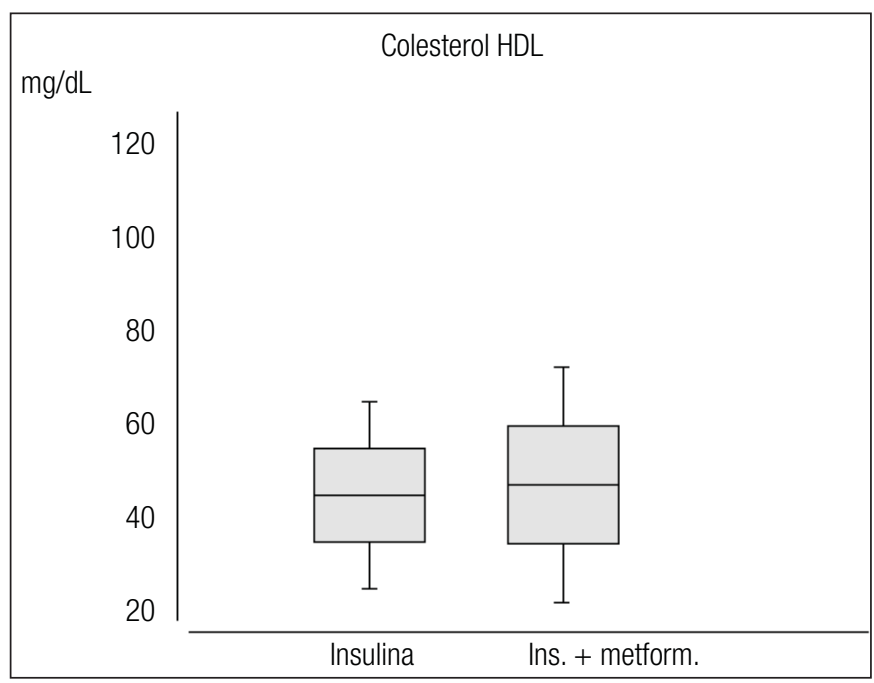

Figura 3: Níveis de colesterol HDL dos pacientes diabéticos tipo 2 em tratamento isolado com insulina e tratamento combinado de insulina+metformina. 
Utilizando a fórmula de Friedewald para o cálculo dos valores médios do LDL, importante determinante de risco cardiovascular, chegou-se ao resultado de $125,4 \mathrm{mg} / \mathrm{dL}$ para o grupo em tratamento isolado com insulina contra 121,94 $\mathrm{mg} / \mathrm{dL}$ para o que estava em terapia combinada; porém, não se pode afirmar com total rigor científico, porque alguns indivíduos apresentaram nível de triglicerídeos superior a $400 \mathrm{mg} / \mathrm{dL}$.

\section{Discussão}

Apesar de muitos pacientes conseguirem sustentar por um longo tempo valores glicêmicos normais, ou próximos do normal, a maioria não mantém o controle glicêmico com a evolução da doença, mesmo com a combinação de vários hipoglicemiantes orais; isto ocorre devido à progressiva deterioração da capacidade de secreção das células beta. Nessa etapa, a introdução de insulina exógena poderia ser realizada concomitantemente à manutenção das ações dos agentes orais, favorecendo o paciente. Entretanto, faltam estudos avaliando o potencial benefício dessa terapia combinada na prevenção das doenças cardiovasculares ${ }^{15}$.

Em um estudo multicêntrico, envolvendo 390 pacientes, realizado em três ambulatórios dos Países Baixos por Kooy et al. ${ }^{16}$, avaliou-se um grupo de portadores de DM2, em uso de insulina e metformina ou insulina e placebo, nos quais foi estabelecido bom controle glicêmico; o primeiro grupo necessitou, em média, de 67 unidades de insulina/dia contra 84 unidades/dia do segundo grupo, para se obter HbA1c de 7,5 e 7,9\%, respectivamente. No presente estudo, também se percebeu necessidade menor na dose de insulina necessária para o controle glicêmico dos indivíduos que utilizavam a terapia combinada com metformina; porém, os pacientes dos grupos analisados não apresentaram controle tão adequado quanto aqueles do estudo holandês, o que pode indicar uma subutilização de insulina em nosso meio.

Em um estudo longitudinal randomizado duplo-cego com duração de 12 meses, realizado por Ponssen et al. ${ }^{17}$, foram abordados 31 pacientes portadores de DM2 (sendo que um se retirou por hipoglicemia); estes receberam tratamentos alternados com insulina e metformina ou insulina e placebo. O grupo em uso de metformina registrou níveis de colesterol total $16,24 \mathrm{mg} / \mathrm{dL}$ e níveis de HbA1c 0,74 ponto percentual mais baixos que o controle. Não se observaram efeitos sobre os valores séricos de HDL ou triglicerídeos. Quanto ao tratamento com a metformina, foram necessárias, em média, 8,69 unidades de insulina a menos por dia; não houve efeitos colaterais importantes.
Avilés-Sant $e t$ al ${ }^{18}$ realizaram um desenho duplo-cego com 43 portadores de DM2 mal controlada em uso de insulina, divididos aleatoriamente para receber placebo ou metformina durante 24 semanas. Os níveis de HbA1c ficaram 1,1 ponto percentual superiores no grupo placebo, sendo que o mesmo ainda precisou de doses maiores de insulina e apresentou valores mais elevados de LDL e colesterol total; HDL e triglicerídeos não variaram.

Avaliando 33 pacientes que estavam em terapia com insulina, Robinson et al. ${ }^{19}$, em um modelo duplo cego randomizado, alternando 12 semanas da associação de metformina com o mesmo tempo de placebo, encontraram níveis de colesterol total em média $23,2 \mathrm{mg} / \mathrm{dL}$ inferiores e ainda valores de HbA1c significativamente favoráveis quando em uso do medicamento, não identificando efeitos importantes em relação a HDL e triglicerídeos.

Em uma revisão abrangendo 41 trabalhos científicos (3.074 pacientes), Wulffelé et al. ${ }^{20}$ coletaram informações sobre o perfil lipídico de portadores de DM2 em tratamento com metformina versus controle. A metformina diminuiu significativamente os níveis plasmáticos de triglicerídeos, em média 11,51 mg/dL ( $\mathrm{p}<0,003)$; colesterol total, 10,05 $\mathrm{mg} / \mathrm{dL}(\mathrm{p}<0,0001) ; \mathrm{LDL}, 8,51 \mathrm{mg} / \mathrm{dL}(\mathrm{p}<0,00001)$ e HbA1c, 0,74 ponto percentual $(\mathrm{p}<0,00001)$; houve ligeira elevação do HDL, que não assumiu importância estatística. Contudo, a redução dos triglicerídeos só foi significativa nos indivíduos em que houve melhor controle glicêmico com a biguanida.

Quanto a este estudo, transversal e não-controlado por placebo, identificou-se no grupo em tratamento com metformina valores de HbA1c 0,79 ponto percentual inferiores aos parâmetros dos pacientes tratados com insulina isolada, atingindo relevância estatística. Houve, nesse mesmo grupo, valores inferiores de colesterol sérico total e mais elevados do HDL, porém sem significado estatístico.

Interessante e antagônica, em relação aos estudos citados nessa discussão que avaliam exclusivamente pacientes em insulinoterapia, foi a redução média de $33,2 \mathrm{mg} / \mathrm{dL}$, estatisticamente significativa, verificada nos valores séricos de triglicerídeos dos indivíduos em uso de metformina. Resultado este que, além disso, extrapola os valores encontrados por Wuffelé et al ${ }^{20} \mathrm{em}$ seu trabalho de revisão, o qual inclui também pacientes que não estavam em tratamento com insulina.

\section{Conclusão}

Os indivíduos portadores de DM2, que estavam em tratamento com insulina associada à metformina, apresentaram 
níveis séricos significativamente inferiores de hemoglobina glicada e triglicerídeos, os quais estão relacionados com menor risco cardiovascular e maior sobrevida, quando comparados aos que usavam apenas insulina.

Em decorrência desses resultados, da baixa renda da maioria dos usuários do SUS e da disponibilidade de metformina na Farmácia Popular do Brasil e gratuitamente nas unidades de atenção primária à saúde, sugerimos empregar, como primeira escolha, tal associação no tratamento de portadores de DM2 que necessitam de insulina exógena, em saúde pública, sendo altamente recomendado iniciar a terapia conjunta, desde que tolerada, para aqueles pacientes que ainda estão em uso de insulina isolada.

\section{Referências}

1. Kahn SE. The relative contributions of insulin resistance and beta-cel dysfunction to the pathophysiology of Type 2 diabetes. Diabetologia. 2003;46(1):3-19.

2. Lu W, Resnick HE, Jablonski KA, Jones KL, Jain AK, Howard WJ, et al. Non-HDL cholesterol as a predictor of cardiovascular disease in type 2 diabetes: the strong heart study. Diabetes Care. 2003;26(1):16-23.

3. Cui Y, Blumenthal RS, Flaws JA, Whiteman MK, Langenberg P, Bachorik PS, et al. Non-high-density lipoprotein cholesterol level as a predictor of cardiovascular disease mortality. Arch Intern Med. 2001;161(11):1413-9.

4. Cullen P. Evidence that triglycerides are an independent coronary heart disease risk factor. Am J Cardiol. 2000;86(9):943-9.

5. Grundy SM, Vega GL, Yuan Z, Battisti WP, Brady WE, Palmisano J. Effectiveness and tolerability of simvastatin plus fenofibrate for combined hyperlipidemia (the SAFARI trial). Am J Cardiol. 2005;95(4):462-8.

6. DaFonseca AL (Dir. científico). DEF 2007/2008: Dicionário de especialidades farmacêuticas. 36ª ed. Rio de Janeiro: Publicações Científicas; 2007.

7. [No authors listed]. Intensive blood-glucose control with sulphonylureas or insulin compared with conventional treatment and risk of complications in patients with type 2 diabetes (UKPDS 33). UK Prospective Diabetes Study (UKPDS) Group. Lancet. 1998;352(9131):837-53.
8. Chandalia HB, Krishnaswamy PR. Glycated Hemoglobin. Curr Sci. 2002;83(12):1522-32.

9. Cheng AYY, Fantus IG. Oral antihyperglycemic therapy for type 2 diabetes mellitus. CMAJ. 2005;172(2):213-26.

10. [No authors listed]. Effect of intensive blood-glucose control with metformin on complications in overweight patients with type 2 diabetes (UKPDS 34). UK Prospective Diabetes Study (UKPDS) Group. Lancet. 1998;352(9131):854-65.

11. Nathan DM, Buse JB, Davidson MB, Ferrannini, E, Holman RR, Sherwin R, Zinman, B. American Diabetes Association ; European Association foro Study of Diabetes. Medical management of hyperglycemia in type 2 diabetes: a consensus algorithm for the initiation and adjustment of therapy: a consensus statement of the American Diabetes Association and the European Association for the Study of Diabetes. Diabetes Care. 2009;32(1):193-203.

12. Buse JB. Combining insulin and oral agents. Am J Med. 2000;108(suppl6a):23S-32S.

13. Brasil. Lista de Medicamentos da Farmácia Popular do Brasil. [citado 2010 ago 6]. Disponível em: http://dtr2002.saude.gov.br/ farmaciapopular/farmacia_popular_arquivos/conteudo/lista.htm.

14. Yki-Järvinen H, Ruysy L, Nikkilä K, Tulokas T, Vanamo R, Heikkillä M. Comparison of bedtime insulin regimens in patients with type 2 diabetes mellitus. Ann Intern Med. 1999;130(5):389-96.

15. Sociedade Brasileira de Diabetes. Diretrizes da Sociedade Brasileira de Diabetes: Tratamento e Acompanhamento do Diabetes mellitus. Rio de Janeiro: Diagraphic. 2008.

16. Kooy A, de Jager J, Lehert P, Bets D, Wulffelé MG, Donker AJM, et al. Long-term effects of metformin on metabolism and microvascular and macrovascular disease in patients with type 2 diabetes mellitus. Arch Intern Med. 2009;169(6):616-25.

17. Ponssen HH, Elte JWF, Lehert P, Schouten JP, Bets D. Combined metformin and insulin therapy for patients with type 2 diabetes mellitus. Clin Ther. 2000;22(6):709-18.

18. Avilés-Santa L, Sinding J, Raskin P. Effects of metformin in patients with poorly controlled, insulin-treated type 2 diabetes mellitus. A randomized, double-blind, placebo-controlled trial. Ann Intern Med. 1999;131(3):182-8.

19. Robinson AC, Burke J, Robinson S, Johnston DG, Elkeles RS. The effects of metformin on glycemic control and serum lipids in insulintreated NIDDM patients with suboptimal metabolic control. Diabetes Care. 1998;21(5):701-5.

20. Wulffelé MG, Kooy A, Zeeuw D, Stehouwer CD, Gansevoort RT. The effect of metformin on blood pressure, plasma cholesterol and triglycerides in type 2 diabetes mellitus: a systematic review. J Intern Med. 2004;256(1):1-14. 